TRANSACTIONS OF THE

AMERICAN MATHEMATICAL SOCIETY

Volume 190,1974

\title{
CLASSICAL QUOTIENT RINGS
}

\author{
BY \\ ROBERT C. SHOCK
}

ABSTRACT. Throughout $R$ is a ring with right singular ideal $Z(R)$. A right ideal $K$ of $R$ is rationally closed if $x^{-1} K=\{y \in R: x y \in K\}$ is not a dense right ideal for all $x \in R-K$. A ring $R$ is a Cl-ring if $R$ is (Goldie) right finite dimensional, $R / Z(R)$ is semiprime, $Z(R)$ is rationally closed, and $Z(R)$ contains no closed uniform right ideals. These rings include the quasi-Frobenius rings as well as the semiprime Goldie rings. The commutative $\mathrm{Cl}$-rings have $\mathrm{Cl}-\mathrm{classical}$ quotient rings. The injective ones are congenerator rings.

In what follows, $R$ is a Cl-ring. A dense right ideal of $R$ contains a right nonzero divisor. If $R$ satisfies the minimum condition on rationally closed right ideals then $R$ has a classical Artinian quotient ring. The complete right quotient ring $Q$ (also called the Johnson-Utumi maximal quotient ring) of $R$ is a $\mathrm{Cl}$-ring. If $R$ has the additional property that $b R$ is dense whenever $b$ is a right nonzero divisor, then $Q$ is classical. If $Q$ is injective, then $Q$ is classical.

1. Introduction. Throughout $Q$ denotes the complete ring of right quotients of $R$ whereas $Z(R)(Z(Q))$ denotes the right singular ideal of $R$ (of $Q$ ). In this paper we look for various conditions on $R$ for $Q$ to be a classical quotient ring. We construct the classical quotient rings via Cl-rings. In a $\mathrm{Cl}$-ring there is an essential direct sum of uniform right ideals $b_{1} R+\cdots+b_{n} R$ such that $b_{1}+\cdots$ $+b_{n}$ is a right nonzero divisor. Furthermore, every dense right ideal contains a right nonzero divisor. This leads to the following theorem:

Theorem 3.2. Suppose that $R$ is a Cl-ring and $b R$ is dense whenever $b$ is a right nonzero divisor of $R$. Then $Q$ is a classical Cl-ring with Jacobson radical $Z(Q)$ and $Q / Z(Q)$ is a completely reducible (semiprime Artinian) classical quotient ring of $R / Z(R)$. The converse bolds.

Immediately, a semiprime Goldie ring (which is a $\mathrm{Cl}$-ring) has a completely reducible classical quotient ring [5]. In a commutative ring, $b R$ is dense whenever $b$ is a nonzero divisor. Therefore, all commutative $\mathrm{Cl}$-rings have classical quotient rings. The abstract states additional applications. (Also cf. Theorem 4.2, Corollary 4.7.)

Received by the editors May 8, 1972.

AMS (MOS) subject class ifications (1970). Primary 16A48, 16A18, 16 A08.

Key words and phrases. Classical quotient ring, complete ring of right quotients, injective, cogenerator, Artinian, quasi-Frobenius ring, rationally closed right ideal. 
The $Q$ of a Cl-ring is a Cl-ring but not conversely. However, if $R / Z(R)$ is semiprime and $Q$ is a $\mathrm{Cl}$-ring then $R$ is also a $\mathrm{Cl}$-ring.

For a nonempty subset $A$ of $R$ we set $r(A)=\{x \in R: a x=0$ for all $a \in A\}$; for $x \in R-A$ we define $x^{-1} A=\{y \in R: x y \in A\}$. Finally, for $b \in R$ we write $r(b)$ instead of $r(\{b\})$ and we equate $b R$ with the principal right ideal generated by $b$.

2. Dense right ideals of $\mathrm{Cl}$-rings. We observe a trivial fact which we use several times.

Remark 2.1. Suppose $a$ and $b$ belong to a uniform right ideal of a ring $R$. If $a b \in R-Z(R)$ then the sum $a R+r(a)$ is direct.

This portion of the paper is devoted to proving Theorem 2.2.

Theorem 2.2. In a Cl-ring $R$ every dense right ideal contains a right nonzero divisor. Furtbermore, so dops every right ideal whose image is essential in $R / Z(R)$.

Proof. For the moment assume that there is an essential direct sum $B$ of uniform right ideals $b_{1} R+\cdots+b_{n} R$ such that $r\left(b_{1}+\cdots+b_{n}\right)=(0)$ and $r\left(b_{i}\right) \cap$ $b_{i} R=(0)$ for $1 \leq i \leq n$. Suppose $L$ is a right ideal with $L \cap b_{i} R \not \subset Z(R)$ for $1 \leq$ $i \leq n$. Hence, $\left(L \cap b_{i} R\right)^{2} \not \subset Z(R)$, and by Remark 1 we choose $x_{i} \in L \cap b_{i} R$ such that $r\left(x_{i}\right) \cap x_{i} R=(0)$. Since $b_{i} R$ and $x_{i} R$ are uniform with nonzero intersection, $r\left(x_{i} b_{i}\right)=r\left(b_{i}\right)$ for $1 \leq i \leq n$. Therefore, $x_{1} b_{1}+\cdots+x_{n} b_{n} \in L$ and is a right nonzero divisor. If $D$ is a dense right ideal and $Z(R)$ is rationally closed then $b_{i}^{-1} D \not \subset Z(R)$ for $1 \leq i \leq n$. Hence, $b_{i} R \cap D \not \subset Z(R)$ and $D$ contains a right nonzero divisor. Next we show that the direct sum $B$ does exist in $R$.

Lemma 2.3. Assume that $R$ is right finite dimensional and $Z(R)$ contains no square of a closed uniform right ideal. Then there is an essential direct sum $b_{1} R+\cdots+b_{k} R$ of uniform right ideals sucb that $r\left(b_{1}+\cdots+b_{k}\right)=(0)$ and $r\left(b_{i}\right) \cap b_{i} R=(0)$ for $1 \leq i \leq k$.

Proof. Let $C$ denote the collection of direct sums of the form $b_{1} R+\cdots+$ $b_{k} R+r(b)$ where $b=b_{1}+\cdots+b_{k}$ and $r\left(b_{i}\right) \cap b_{i} R=(0)$ for $1 \leq i \leq k$. Remark 2.1 shows that $C$ is nonempty. Let $b_{1} R+\cdots+b_{k} R+r(b)$ be a maximal element of $C$ in that if $c_{1} R+\cdots+c_{p} R+r(c)$ is an element of $C$ then $p \leq k$. Equate $B=b_{1} R+\cdots+b_{k} R$. If $r(b)=(0)$, then $b R$ is essential and we are finished. Assume that $r(b) \neq(0)$ and by Zorn's lemma choose a closed uniform right ideal $A$ subject to $A \cap r(b) \neq(0)$ and $A \cap B=(0)$. Since $A^{2}-Z(R)$ is nonempty by hypothesis, we choose $a \in A$ such that $r(a) \cap a R=(0)$; see Remark 2.1. It suffices to show that $(B+a R) \cap r(a+b)=(0)$ for then $a+r(b)$ is not maximal, a contradiction. Suppose $x=b^{\prime}+a^{\prime}$ with $b^{\prime} \in B$ and $a^{\prime} \in B R$ and $b x=a x=0$. If $a^{\prime}=0$ then $b x=b b^{\prime}=0$ and $b^{\prime}=0=x$ since $r(b) \cap B=(0)$. If $a^{\prime} \neq 0$ then $a^{\prime} R$ $\cap r(b) \neq(0)$; recall the uniform right ideal $A$ has nonzero intersection with $r(b)$. 
There is $z \in R$ such that $0 \neq x z=b^{\prime} z+a^{\prime} z$ with $a^{\prime} z \in r(b)-(0)$. Hence, $b x z=$ $b b^{\prime} z=0$ implies $b^{\prime} z=0$. Again $a x z=a a^{\prime} z$ implies $a^{\prime} z=0$. Therefore, $x z=0$, a contradiction. We conclude that $x=0$ which completes the lemma.

Clearly, a Cl-ring satisfies the hypothesis of Lemma 2.3 which completes the proof of Theorem 2.2.

3. A classical quotient theorem for Cl-rings. It is easy to see that if each dense right ideal of $\boldsymbol{R}$ contains a nonzero divisor and nonzero divisors are invertible in $Q$, then $Q$ is a classical quotient ring with no proper dense right ideals; the converse holds. We set up conditions on $R$ for this to occur.

Remark 3.1. Assume that $b$ is a right nonzero divisor of $R$. Then $b R$ is dense if and only if $b$ is invertible in $Q$.

To see this, the map $b r$ to $r$ implies that $q b=1$ for an appropriate $q \in Q$. Since $b R$ is essential, $r(q)=(0)$ and $b q=1$ which proves the remark.

Theorem 3.2. Assume that $R$ is a Cl-ring and $b R$ is dense whenever $b$ is a rigbt nonzero divisor of $R$. Then $Q$ is a Cl-classical quotient ring of $R$ and the Jacobson radical of $Q$ is $Z(Q)$. Furthermore, $Q / Z(Q)$ is a completely reducible classical quotient ring of $R / Z(R)$. The converse bolds.

Proof. From Remark 3.1 and Theorem 2.2 we see that $Q$ is a classical quotient ring and that $Q / Z(Q)$ contains no proper essential right ideals. Therefore, $Q / Z(Q)$ is a completely reducible ring. If $a b^{-1} \in Z(Q)$ with $r(b)=(0)$ and $a \in R$ then $\left(1-a b^{-1}\right) b$ is a right nonzero divisor of $R$ and is invertible in $Q$. Hence, the Jacobson radical of $Q$ is $Z(Q)$. For the converse it is easy to see that $R$ is a Cl-ring. If $b \in R$ with $r(b)=(0)$ then the right nonzero divisor $b+Z(R)$ is invertible in $Q / Z(Q)$. Since $Z(Q)$ is the Jacobson radical it follows that $b$ is invertible in $Q$. Remark 3.1 completes the implication.

Corollary 3.3 [5]. If $R$ is a semiprime Goldie ring then $R$ bas a classical completely reducible quotient ring. The converse bolds.

Proof. The proof is clear.

4. Applications. We recall some known relationships between $R$ and $Q$. Clearly, $Z(Q) \cap R=Z(R)$. Closed right ideals are rationally closed. Recall, a right ideal $K$ is rationally closed if $x^{-1} K=\{y \in R: x y \in K\}$ is not a dense right ideal. An equivalent statement for a right ideal $K$ to be rationally closed is that the injective hull of $R$ contains a subset $S$ such that $K=\{y \in R: s y=0$ for all $s \in S\}$ [18]. It follows that the maximum (minimum) condition on rationally closed right ideals as well as the finite dimensional property passes from $R$ to $Q$ and conversely. The $\mathrm{Cl}$-property passes from $R$ to $Q$.

Theorem 4.1. The $Q$ of a Cl-ring is a Cl-ring. If $R / Z(R)$ is semiprime and $Q$ is a Cl-ring then $R$ is a Cl-ring. 
Proof. Let $R$ be a Cl-ring and let $q \in Q-Z(Q)$. If $q^{-1} Z(Q)$ were dense then it would contain a right nonzero divisor $b$ by Theorem 2.2 and $q b \in Z(Q)$. However, $r(q b)=b R \cap A(q)$ and $q b \in R-Z(Q)$ because $r(q)$ is not essential. We have a contradiction and conclude that $Z(Q)$ is rationally closed. If $K$ is a uniform closed right ideal of $Q$, then $K \cap R$ is closed and $K \cap R \not \subset Z(R)$; hence, $K \cap Q \not \subset Z(Q)$. For the next implication, $Z(R)=R \cap Z(Q)$ is rationally closed since $Z(Q)$ is. Let $A$ denote a closed uniform right ideal of $R$ whereas $A^{\prime}$ denotes a closed uniform right ideal of $Q$ containing $A$. Then $A^{\prime} \not \subset Z(Q)$ implies $A^{\prime} \cap R=A \not \subset Z(R)$ which completes the proof.

Although $Q$ may be a Cl-ring, $R$ need not be. Let $R$ denote the upper triangular $2 \times 2$ matrices over a field. Then $Q$ is completely reducible and $Z(R)=$ (0) yet $R$ is not semiprime.

We apply Theorem 2.2 to self-injective rings. A ring $R$ is a self-injective cogenerator if $R_{R}$ is injective and $R_{R}$ is a cogenerator in the category of unital right $R$-modules [10]. These rings are finite dimensional and properly contain the quasi-Frobenius rings [13].

Suppose $R$ is a finite dimensional self-injective ring. Then $R$ is a cogenerator ring if and only if $Z(R)$ is rationally closed [19]. Hence, a self-injective ring is a Cl-ring if and onlyif it is a cogenerator ring.

A ring $R$ has the dense extension property if each map from a right ideal of $R$ into $R$ can be extended to a dense right ideal. The complete ring of right quotients $Q$ of $R$ is self-injective if and only if $R$ has the dense extension property. This is an immediate consequence of the Lambek-Gabriel characterization as in [3] and [4].

Theorem 4.2. A Cl-ring with the dense extension property has a classical quotient ring which is a self-injective cogenerator ring. The converse bolds.

Proof. The $Q$ of a ring $R$ with the above properties is an injective $\mathrm{Cl}$-ring. If $b \in R$ with $r(b)=(0)$ then $b Q$ is an essential injective $Q$-submodule of $Q$ and thus $b Q=Q$. Therefore, right nonzero divisors are invertible in $Q$. From Theorem 3.2 and Remark 3.1, $Q$ is classical. The converse is clear.

The corollary below is a special case of Theorem 5.3 of [18].

Corollary 4.3. If $R / Z(R)$ is semiprime and $Q$ is a self-injective cogenerator ring then $Q$ is a classical quotient ring.

Proof. The result is clear.

Our next corollary is due to Johnson [8, Theorem 4.4] and Sandomierski [16, Theorem 1.6]. 
Corollary 4.4. If $R$ is semiprime and $Q$ is completely reducible then $Q$ is a classical quotient ring.

Proof. The proof is clear.

A solid Goldie ring is one with the maximum condition on rationally closed right ideals [6]. The solid Goldie rings are Goldie rings but not conversely [18]. The injective ones are quasi-Frobenius [1]. The next corollary was proven independently by Shock [18] (announced in [17]) and by Tachikawa [20].

Corollary 4.5. A ring $R$ bas a classical quasi-Frobenius quotient ring if and only if $R$ is a solid Goldie with the dense extension property and the prime radical of $R$ is the right singular ideal.

Proof. Recall $R$ is solid Goldie if and only if $Q$ is. If $Q$ is quasi-Frobenius then $Z(Q) \cap R=Z(R)$ and $Z(R)$ is nilpotent. The proof is clear.

The dual notion of the solid Goldie ring is the ring with the minimum condition on rationally closed right ideals. Assume that $R$ has this latter property. If $R$ is singular $(Z(R)=(0))$, then the rationally closed right ideals are closed and the property coincides with the finite dimensional property. The other extreme is that $R$ contains no proper dense right ideal and all right ideals are rationally closed; in this case $R$ is Artinian. These rings contain the Artinian rings. The injective ones are quasi-Frobenius. In an Artinian ring a nonzero divisor is invertible. In $R$ a nonzero divisor is invertible in $Q$ as we see below.

Theorem 4.6. In a ring with the minimum condition on rationally closed right ideals, a right nonzero divisor is invertible in $Q$.

Proof. Suppose that $b \in R$ and $r(b)=(0)$. It suffices to show that $b R$ is a dense right ideal; see Remark 3.1. Assume that $b R \supset b^{2} R \supset b^{3} R \supset \ldots$ is a strictly decreasing sequence, otherwise $b$ is invertible in $R$. Clearly, $\left(b^{n}\right)^{-1} b^{n+1} R=b R$. If $b R$ were not dense then the rational closure of $b^{n} R$ would properly contain the rational closure of $b^{n+1} R$ (cf. [18, Lemma 3.1(2)]). We would then have a strictly decreasing sequence of rationally closed right ideals, a contradiction. Therefore, $b R$ is dense.

Corollary 4.7. A Cl-ring with the minimum condition on rationally closed right ideals bas a classical Cl-Artinian quotient ring. The converse bolds.

Proof. The proof is clear.

We state the dual to Corollary 4.5 .

Corollary 4.8. A ring $R$ bas a classical quasi-Frobenius quotient ring if and only if $R$ satisfies the dense extension property and $R$ satisfies the minimum condition on rationally closed right ideals and the prime radical is the right singular ideal. 
Proof. The proof is clear.

\section{REFERENCES}

1. C. Faith. Rings with ascending condition on annihilators, Nagoya Math. J. 27 (1966), 179-191. MR 33 \#1328.

2. C. Faith and E. Walker, Direct-sum representations of injective modules, J. Algebra 5 (1967), 203-221. MR 34 \#7575.

3. G. D. Findlay and J. Lambek, $A$ generalized ring of quotients. I, II, Canad. Math. Bull. 1 (1958), 77-85; 155-167. MR 20 \#888.

4. P. Gabriel, Des catégories abéliennes, Bull. Soc. Math. France 90 (1962), 323448. MR 38 \#1144.

5. A. W. Goldie, Semiprime rings with the maximum condition, Proc. London Math. Soc (3) 10 (1960), 201-220. MR 22 \#2627.

6. J. P. Jans, On orders in quasi-Frobenius rings, J. Algebra 7 (1967), 35-43. MR 35 \#2925.

7. R. Johnson, Extended centralizer of a ring over a module, Proc. Amer. Math. Soc. 2 (1951), 891-895. MR 13, 618.

8. - Quotient rings of rings with zero singular ideal, Pacific J. Math. 11 (1961), 1385-1392. MR 26 \#1331.

9. J. Lambek, Lectures on rings and modules, Blaisdell, Waltham, Mass, 1966. MR 34 \#5857.

10. K. Morita, Duality for modules and its applications to the theory of rings with minimum conditions, Sci. Rep. Tokyo Kyoiku Daigaku Sect. A 6 (1958), 83-142. MR 20 \#3183.

11. T. Nakayama, On Frobeniusean algebras. I, Ann. of Math. 40 (1939), 611-633. MR 1, 3.

12. A. C. Mewborn and C. N. Winton, Orders in self-injective semiperfect rings, J. Algebra 13 (1969), 5-9. MR 39 \#6925.

13. B. Osofsky, A generalization of quasi-Frobenius rings, J. Algebra 4 (1966), 373-387. MR $34 \# 4305$.

14. J. C. Robson, Artinian quotient rings, Proc. London Math. Soc. (3) 17 (1967), 600-616. MR 36 \#199.

15. L. W. Small, Orders in Artinian rings, J. Algebra 4 (1966), 13-41. MR 34 \#199.

16. F. Sandomierski, Semisimple maximal quotient rings, Trans. Amer. Math. Soc. 128 (1967), 112-120. MR 35 \#5473.

17. R. C. Shock, Right orders in self injective rings, Notices Amer. Math. Soc. 17 (1970), p. 561. Abstract \#70T-A76.

18. —_, Injectivity, annihilators, and orders, J. Algebra 19 (1971), 96-103. MR 43 \#4861.

19. - Orders in self-injective cogenerator rings, Proc. Amer. Math. Soc. 35 (1972), 393-398.

20. H. Tachikawa, Localization and Artinian quotient rings, Math. Z. 119 (1971), 239-253.

DEPARTMENT OF MATHEMATICS, SOUTHERN ILLINOIS UNIVERSITY, CARBONDALE, ILLINOIS 62901 